\title{
Characterization of human periodontal ligament cells cultured on three-dimensional biphasic calcium phosphate scaffolds in the presence and absence of $L$-ascorbic acid, dexamethasone and $\beta$-glycerophosphate in vitro
}

\author{
SHAOFENG AN, YAN GAO and JUNQI LING
}

\begin{abstract}
Department of Operative Dentistry and Endodontics, Guanghua School of Stomatology, Hospital of Stomatology, Sun Yat-sen University; Guangdong Provincial Key Laboratory of Stomatology, Guangzhou, Guangdong 510055, P.R. China
\end{abstract}

Received December 19, 2014; Accepted August 3, 2015

DOI: $10.3892 /$ etm.2015.2706

\begin{abstract}
The aim of this study was to evaluate the effect of porous biphasic calcium phosphate (BCP) scaffolds on the proliferation and osteoblastic differentiation of human periodontal ligament cells (hPDLCs) in the presence and absence of osteogenic inducer (L-ascorbic acid, dexamethasone and $\beta$-glycerophosphate). The cell growth within the scaffolds in the absence of osteogenic inducers was studied by cell counting kit-8 (CCK-8) assay and scanning electron microscopy (SEM). Alkaline phosphatase (ALP) activity and osteoblastic differentiation markers of hPDLCs in BCP scaffolds were examined in the presence and absence of osteogenic inducers. The cell number of hPDLCs in the BCP scaffolds was less than that of hPDLCs cultured in microplates (control). SEM images showed that cells successfully adhered to the BCP scaffolds and spread amongst the pores; they also produced abundant extracellular cell matrix. In the presence and absence of osteogenic inducers, the ALP activity of hPDLCs within BCP scaffolds was suppressed in varying degrees at all time-points. In the absence of osteogenic inducers, hPDLCs in BCP scaffolds express significant higher levels of osteopontin (OPN) mRNA than the control, and there were no significant differences for Runx2 and osteocalcin (OCN) mRNA levels compared with those cultured in microplates. In the presence of osteogenic inducers, Runx 2 expression levels were significantly higher than those in control. OPN and OCN mRNA levels were downregulated slightly. Three-dimensional porous
\end{abstract}

Correspondence to: Professor Junqi Ling, Department of Operative Dentistry and Endodontics, Guanghua School of Stomatology, Hospital of Stomatology, Sun Yat-sen University; Guangdong Provincial Key Laboratory of Stomatology, 56 Ling Yuan Xi Road, Guangzhou, Guangdong 510055, P.R. China E-mail: lingjq@mail.sysu.edu.cn

Key words: human periodontal ligament cells, osteoblastic differentiation, biphasic calcium phosphates, scaffolds, biocompatibility
BCP scaffolds are able to stimulate the osteoblastic differentiation of hPDLCs in the presence and absence of osteogenic inducer and may be capable of supporting hPDLC-mediated bone formation.

\section{Introduction}

Periodontitis is a common human infectious disease characterized by loss of connective tissue and bone support $(1,2)$. If left untreated, uncontrolled periodontal tissue destruction eventually leads to tooth migration, increased mobility and tooth loss, impairing chewing function, phonetics and esthetics (3). The ultimate goal of periodontal treatment is to restore the structure and function of the destroyed hard (alveolar bone and cementum) and soft (gingival attachment and periodontal ligament) connective tissues using periodontal-regenerative techniques (4).

Over the last few decades, several therapeutic techniques have been developed for periodontal regeneration, including soft or hard tissue replacement grafts, root surface planning, guided tissue/bone regeneration (GTR/GBR) and the delivery of growth factors or gene therapies $(3,5)$. Recent advances in progenitor cell biology and tissue engineering have enabled the application of cell-based periodontal regeneration using various approaches and principles. Since human periodontal ligament cells (hPDLCs) have multipotential characteristics, the cells are regarded as useful sources for the regeneration of periodontal tissues containing bone, cementum and periodontal ligament (6-8).

Calcium phosphate bioceramics have been used as a bone substitutes to provide a scaffold for cell migration and rapid bone formation because they have unique characteristics compared with other biomaterials. These bioceramics have a close chemical and crystal resemblance to bone mineral and are widely used in orthopedic and dental applications (9). Calcium phosphate scaffolds vary by their physicochemical properties. There are two widely used forms of calcium phosphate ceramics: Hydroxyapatite $\left(\mathrm{HA}, \mathrm{Ca}_{10}\left[\mathrm{PO}_{4}\right]_{6}[\mathrm{OH}]_{2}\right)$ and tricalcium phosphate (TCP, $\left.\mathrm{Ca}_{3}\left[\mathrm{PO}_{4}\right]_{2}\right)(9,10)$. HA scaffolds are strongly osteoconductive but minimally osteogenic, and have been used to repair cranial-facial and other bone defects. 
However, HA is quite brittle and resorbs slowly when used alone (11). $\beta$-tricalcium phosphate $(\beta$-TCP) was also utilized in bone regeneration in vitro and in vivo duo to its osteoconductivity and bioresorbability (11-13). A major drawback of $\beta$-TCP scaffolds is that the in vivo bone resorption rate of the scaffold exceeds that of the formation of native bone (11). In order to reduce the independent disadvantages of HA and $\beta$-TCP, which are largely in opposition to one another, scaffolds composed of these two compounds in various combinations and ratios have been designed.

Biphasic calcium phosphate (BCP) scaffolds consist of variable amounts of HA and $\beta$-TCP and their capacity to release calcium and phosphate ions into the local microenvironment may also vary (11). A number of studies have indicated that BCP scaffolds are superior to HA scaffolds and even native bone mineral in mediating bone regeneration $(11,14,15)$. However, the majority of the aforementioned knowledge has been obtained using the organic factors L-ascorbic acid (L-aa), dexamethasone (Dex) and $\beta$-glycerophosphate ( $\beta$-GP), which are osteogenic inducers or osteogenic media in vitro $(16,17)$. However, these factors are not expected to be useful in vivo. In addition, to our knowledge, few studies have investigated whether BCP exerts intrinsic inductive capacity on the osteoblastic differentiation of human periodontal ligament cells (hPDLCs) in the presence and absence of osteogenic inducers. Thus, the objectives of the present study were to determine the biological properties of BCP scaffolds on hPDLCs, and to evaluate their effect on the osteoblastic differentiation of hPDLCs in the presence and absence of osteogenic inducers.

\section{Materials and methods}

Cell culture. The experimental protocol was approved by the Ethics Committee of Sun Yat-Sen University (Guangzhou, China), and informed consent was obtained from all the subjects. The hPDLCs were isolated as previously described (18). In short, fresh periodontal ligament (PDL) tissues were removed from the middle-third of the root and minced, and then were plated in fresh Dulbecco's modified Eagle's medium (DMEM/high glucose, HyClone; GE Healthcare Life Sciences, Logan, UT, USA) containing $20 \%$ fetal bovine serum (FBS; Biological Industries, Kibbutz Beit Haemek, Israel) and 2\% (v/v) penicillin/streptomycin (Invitrogen Life Technologies, Carlsbad, CA, USA). An explant culture method was used to enable cells to migrate out of the tissue samples in a humidified atmosphere of $5 \% \mathrm{CO}_{2}$ at $37^{\circ} \mathrm{C}$. Once $\sim 80 \%$ confluence was met, the cell cultures were collected by trypsinization ( $0.25 \%$ trypsin/EDTA; Invitrogen Life Technologies) and passaged for future studies. Cells at the fifth passage were used in subsequent experiments.

Osteoblastic differentiation of hPDLCs (Alizarin red $S$ staining). The fifth passage of hPDLCs was seeded in a 6-well plate, and cultured in DMEM growth culture medium (GM) supplemented with $10 \%$ FBS and $2 \%$ penicillin/streptomycin (in the absence of osteogenic inducers) or in osteogenic culture medium (OM) with $10 \%$ FBS, $10 \mathrm{mM} \beta$-GP, $10^{-8} \mathrm{M}$ Dex, $50 \mu \mathrm{g} / \mathrm{ml} \mathrm{L-aa} \mathrm{(containing} \mathrm{osteogenic} \mathrm{inducers).} \mathrm{After} 7$ and 14 days of culture, the process of mineralized matrix deposition formation was observed using an Alizarin red S staining kit (GenMed, Shanghai, China) according to the manufacturer's instructions.

Scaffold preparation and cell seeding. BCP scaffolds were supplied by the National Engineering Research Center for Biomaterials (Chengdu, China). The BCP ceramics consist of $70 \%$ HA and $30 \% \beta$-TCP. The porosities of the ceramics were $\sim 70 \%$. Prior to use, all scaffolds were sterilized for $15 \mathrm{~min}$ $\left(121^{\circ} \mathrm{C}, 15\right.$ bar pressure) by autoclaving. Prior to cell seeding, all scaffolds were soaked in DMEM supplemented with $10 \%$ FBS and $2 \%$ penicillin/streptomycin at $37^{\circ} \mathrm{C}$ for $4 \mathrm{~h}$. Then, the hPDLCs were seeded on the scaffolds. The cell-scaffold complexes were maintained in 24-well culture plates and divided into two groups. One of the groups was used for cell proliferation assay and scanning electron microscopy (SEM) observation. The scaffolds were incubated with a suspension of $1 \times 10^{5}$ cells $/ \mathrm{ml}$, using $20 \mu \mathrm{l}$ cell suspension per scaffold. The cell-scaffold constructs were then cultured in the GM. The other group was used for osteoblastic differentiaton analysis. The scaffolds were incubating with a suspension of $3 \times 10^{6}$ cells $/ \mathrm{ml}$, using $100 \mu \mathrm{l}$ cell suspension per scaffold. The cell-scaffold complexes were then cultured in the OM. Culture medium was refreshed at 3-day intervals. hPDLCs cultured within plastic culture plates served as the control.

Cell proliferation assay. The proliferation rates of hPDLCs within BCP scaffolds were measured in the GM using a Cell Counting kit-8 (CCK-8; Beyotime Institute of Biotechnology, Shanghai, China). In brief, the cells were seeded on scaffolds in 24-well plates at $2 \times 10^{3}$ cells/per scaffold, with four repeats for each group. Cells were removed from the scaffolds through trypsinization and replaced with $100 \mu \mathrm{l}$ culture medium on days $1,2,3,4,5$ and 6 . The cultures were then transferred to 96 -well plates. To each well was added $10 \mu \mathrm{l}$ CCK-8 solution. The plates were incubated at $37^{\circ} \mathrm{C}$ for $4 \mathrm{~h}$ in a humidified atmosphere of $5 \% \mathrm{CO}_{2}$. The absorbance of the supernatant was measured with a microplate meter (Infinite 200; Tecan, Männedorf, Switzerland) at $490 \mathrm{~nm}$. Non-seeded scaffolds in the same medium were used as a negative control.

SEM analysis. On days 3 and 7 after cell seeding, cell-scaffold complexes were washed with phosphate-buffered saline (PBS) twice and fixed in $2.5 \%$ glutaraldehyde for $4 \mathrm{~h}$. Then, the samples were dehydrated in a graded ethanol series and air-dried in tetramethylsilane (Merck Millipore, Darmstadt, Germany). After gold sputtering, the specimens were examined with a Quanta 200 scanning electron microscope (FEI, Hillsboro, OR, USA). As a blank control, BCP scaffolds were investigated, which were incubated in the same culture medium but without cells.

Alkaline phosphatase (ALP) activity. After 3 and 7 days of culture, ALP activity was measured by an ALP activity assay kit (Nanjing Jiancheng Biotechnology Co. Ltd., Nanjing, China) following the manufacturer's instructions. All analyses were carried out at three separate preparations of experiments. In brief, the adherent cells were removed from the scaffolds through trypsinization and lysed with PBS, followed by addition of a cell lysis buffer containing $0.1 \%$ Triton X-100 to the samples, which were then subjected to three freeze-thaw 
Table I. Primer sequences used for reverse transcription-quantitative polymerase chain reaction.

\begin{tabular}{lll}
\hline Genes & Direction & \multicolumn{1}{c}{ Primer } \\
\hline Runx2 & Forward & Size $(\mathrm{bp})$ \\
& Reverse & CCAACCCACGAATGCACTATC \\
Osteopontin & Forward & TAGTGAGTGGTGGCGGACATAC \\
Osteocalcin & Reverse & GCCGAGGTGATAGTGTGGTT \\
& Forward & TGAGGTGATGTCCTCGTCTG \\
GAPDH & Reverse & GCCTCCTCGCCCTATTGGC \\
& Forward & CATGTTCCAATATGATTCCACC \\
\hline
\end{tabular}

GAPDH, glyceraldehyde 3-phosphate dehydrogenase.

cycles. Aliquots (50 $\mu \mathrm{l}$ each well) of these supernatants were placed into 24-well plates to which $50 \mu \mathrm{l}$ ALP substrate solution ( $2 \mathrm{mM} \mathrm{MgCl}_{2}$ and $16 \mathrm{mM}$-nitrophenyl phosphate) was added. Following incubation at $37^{\circ} \mathrm{C}$ for $30 \mathrm{~min}$, the reaction was stopped by the addition of $50 \mu 10.2 \mathrm{M} \mathrm{NaOH}$, and the liberated $p$-nitrophenol was measured on the Tecan microplate reader at $520 \mathrm{~nm}$.

Reverse transcription-quantitative polymerase chain reaction $(R T-q P C R)$. A total of $3 \times 10^{5}$ cells were seeded into each scaffold in 24-well plastic culture plates. The expression of Runx2, osteopontin (OPN) and osteocalcin (OCN) mRNA was determined by RT-qPCR on day 21 after initial seeding. The total cell RNA from each scaffold $(n=3)$ was harvested using TRIzol reagent (Invitrogen Life Technologies). Total RNA was used as template for the synthesis of cDNA with Oligo(dT) and RevertAid ${ }^{\mathrm{TM}}$ M-MuLV Reverse Transcriptase (MBI/Fermentas, Thermo Fisher Scientific, Pittsburgh, PA, USA). The subsequent PCR amplification reaction was conducted Taq polymerase (Invitrogen Life Technologies) and specific primers. Each PCR was duplicated with the same amount of total RNA. Relative gene expression levels of Runx2, OPN, and OCN were normalized to the expression of the reference gene glyceraldehyde 3-phosphate dehydrogenase (GAPDH). Primers for the selected genes are listed in Table I.

Statistical analysis. All experiments were performed three times, with each treatment conducted in triplicate. Means and standard deviations were calculated, and the statistical significance of differences between groups was examined by one-way analysis of variance. SPSS software, version 17.0 (SPSS, Inc., Chicago, IL, USA) was employed for all statistical analysis and differences were considered significant if $\mathrm{P}<0.05$.

\section{Results}

Characterization and osteogenic differentiation of hPDLCs. The isolated hPDLCs had typical fibroblastic morphology with triangular, spindle-like, cuboid and short fusiform shapes (Fig. 1A). The Alizarin red staining results indicated that the differentiation of hPDLCs into osteoblast-like cells and the deposition of calcium occurred only in the presence of $\beta$-GP,
Dex and L-aa, and that prolonged culturing resulted in the production of larger mineralization nodules (Fig. 1B).

Cell proliferation. The cell number in cell-scaffold complexes from day 1 to day 6 was lower than that in the control group (monolayer cultures in microplates). There was statistically significant difference in cell proliferation levels between the experimental groups and the control at days 2, 3 and 4 $(\mathrm{P}<0.05)$. Following 4 days of culture in the GM, however, the cell number within BCP scaffolds was only slightly lower than that in the control ( $\mathrm{P}>0.05$; Fig. 2).

Observation by SEM. At day 3 after seeding, hPDLCs successfully adhered to the BCP scaffolds, proliferating and spreading among the pores within the three-dimensional network of the scaffold. SEM images demonstrated that the majority of the cells were polygonal in shape, and the number of cells adhering to the scaffold surface was relatively low (Fig. 3A and B). At day 7, large numbers of hPDLCs were observed to have adhered to and proliferated significantly within the $\mathrm{BCP}$ scaffolds. There were numerous filamentous extracellular matrices visible on the surface of the cells (Fig. 3C and D).

ALP activity. In the GM and in the OM, the ALP activity gradually increased from day 3 to day 7 . The ALP activity of cells cultured on a BCP scaffold exhibited no difference from that of the control cells, cultured in microplates, at day 3 in either medium. However, the ALP activity of hPDLCs within the scaffolds was suppressed significantly compared with the activity of the control hPDLCs at 7 day, and the extent of the suppression differed between in the GM and $\mathrm{OM}(\mathrm{P}<0.05$; Fig. 4).

BCP scaffolds affect the osteoblastic differentiation of hPDLCs. Following 21 days of the induction of osteogenic differentiation, compared with the hPDLCs cultured in plastic culture plates, hPDLCs within BCP scaffolds showed significantly higher levels of Runx2 mRNA in the $\mathrm{OM}(\mathrm{P}<0.01)$, and OPN in the GM ( $\mathrm{P}<0.001$; (Fig. 5A and B). Runx 2 mRNA levels in the GM and OPN mRNA levels in the OM were also downregulated, but there was no significant difference in expression level between the experimental group and the 
A

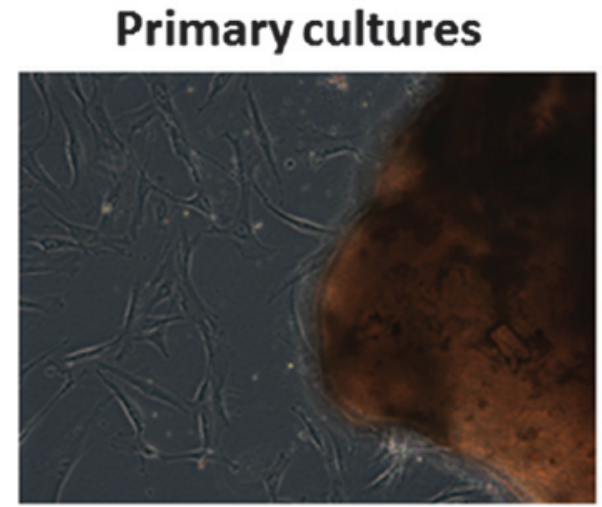

B
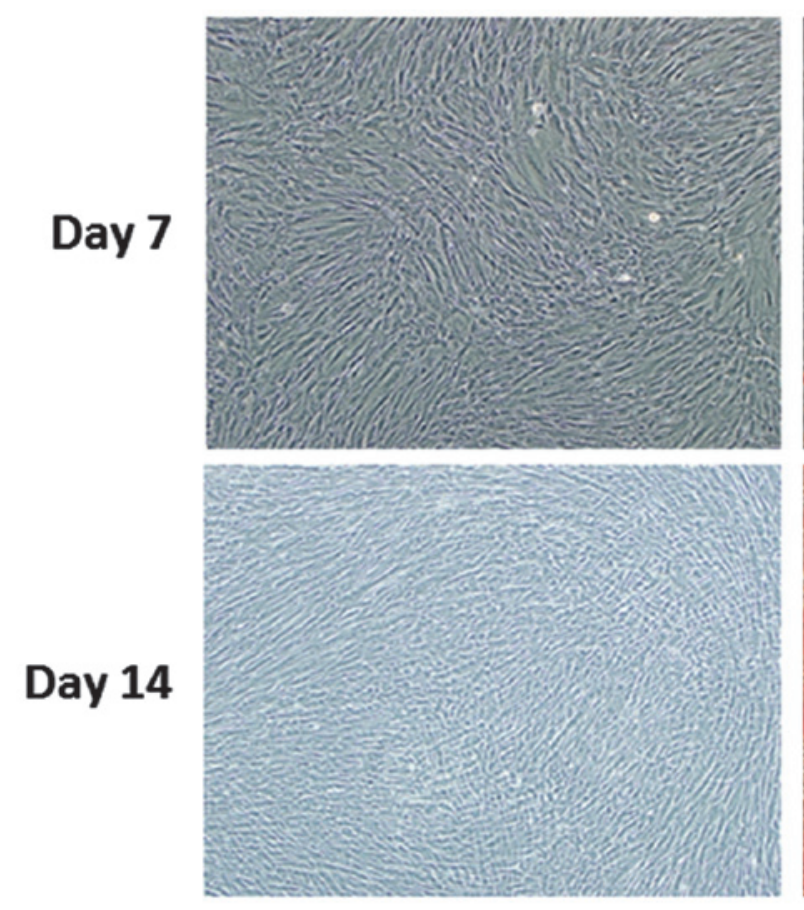

Subcultures

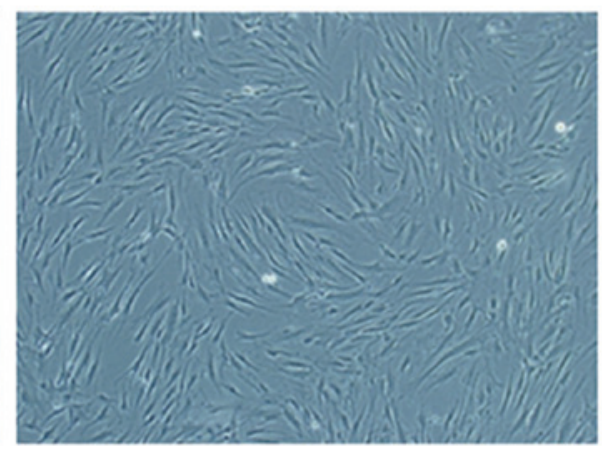

OM
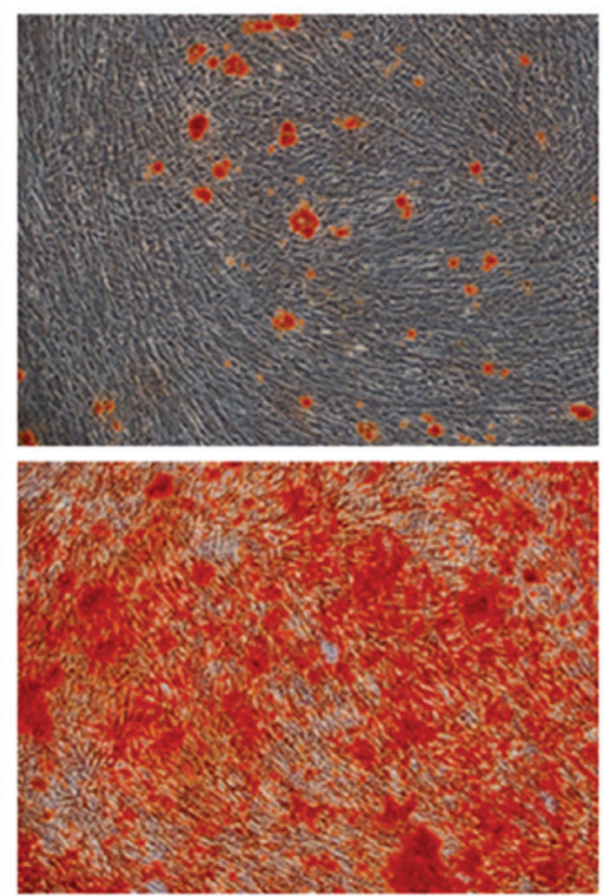

Figure 1. Cell morphology and evaluation of osteogenic differentiation capacity in human periodontal ligament cells (hPDLCs). (A) Primary cultures and subcultures. The isolated cells had typical fibroblastic morphology with triangular, spindle-like and cubic shapes. (B) Evaluation of the osteogenic differentiation of hPDLCs using Alizarin red staining at days 7 and 14. In the absence of osteogenic inducers (growth medium, GM), there wasa no formation of mineralized nodes. In the presence of osteogenic induers (osteogenic medium, OM), hPDLCs were induced into osteoblast-like cells and calcium deposition was detected. Osteogenic culturing of the cells for longer periods of time resulted in more mineralization (magnification, x100).

control (Fig. 5A and B). OCN mRNA expression levels in the $\mathrm{GM}$ and $\mathrm{OM}$ were downregulated in the BCP scaffolds but also exhibited no significant changes ( $\mathrm{P}>0.05$; Fig. $5 \mathrm{C})$.

\section{Discussion}

Ideal biomedical scaffolds should be biocompatible and nontoxic. One of the first steps in the development of a novel scaffold is the evaluation of its cytotoxicity (3). In the present study, SEM demonstrated that hPDLCs covered most of the outer surfaces of the BCP scaffolds by spreading themselves, and also produced abundant extracellular cell matrix (ECM). ECM plays a very important role in the adhesion, proliferation, and differentiation of cells in the surface of the materials $(7,9)$. Such a response indicates good cytocompatibility and close interaction of the scaffolds with hPDLCs in vitro. CCK-8 anal-

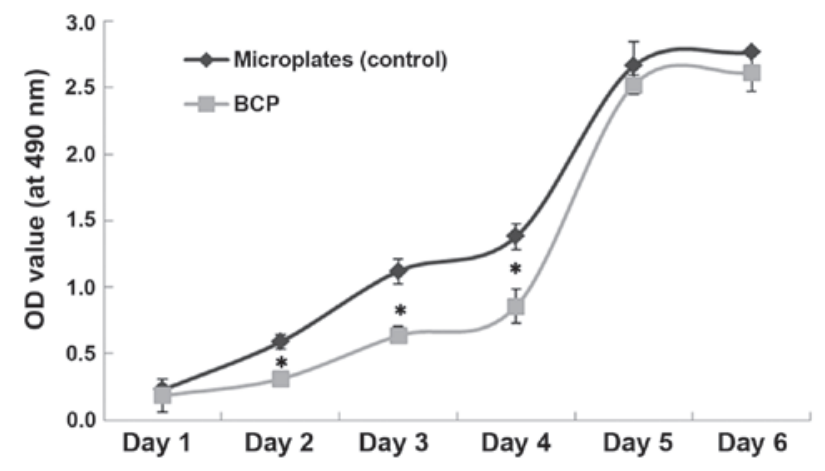

Figure 2. Proliferation of human periodontal ligament cells (hPDLCs) in biphasic calcium phosphate (BCP). The rate of hPDL proliferation in BCP was inhibited significantly compared with control (monolayer cultures in a microplates) at days 2, 3 and 4. However, at days 5 and 6 , the proliferation rate of hPDLCs within the BCP was slightly lower than that in the control. ${ }^{*} \mathrm{P}<0.05$ vs. the control. OD, optical density. 

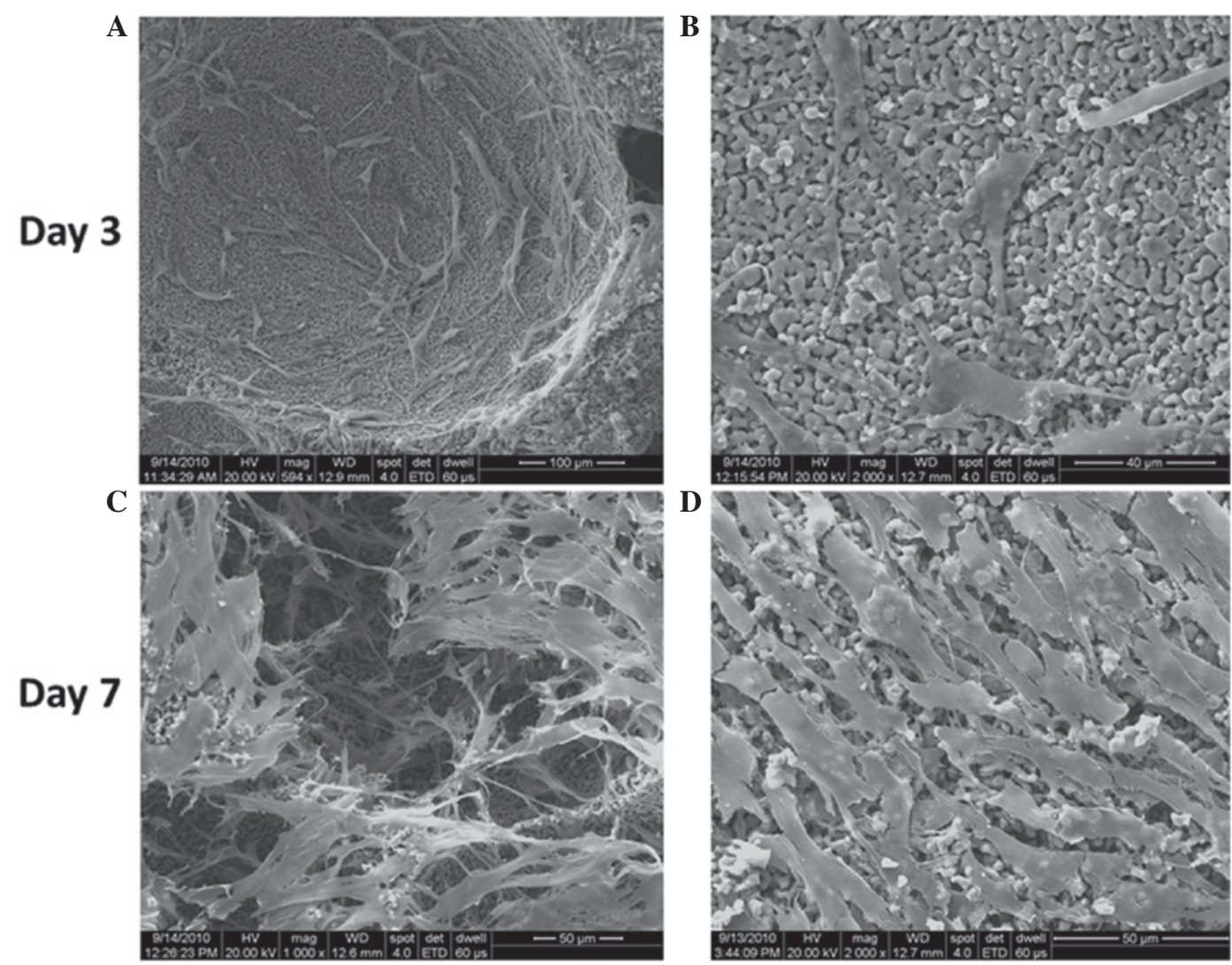

Figure 3. Scanning electron microscopy of the growth of human periodontal ligament cells (hPDLCs) within biphasic calcium phosphate (BCP) scaffolds. (A and B) At day 3, images showed that cells successfully adhered to the BCP scaffolds, were spread over the material surfaces and had migrated inside the pores. The majority of the cells were polygonal in shape. The number of cells adhering on the scaffold surface was relatively low. (C and D) At day 7, large amounts of hPDLCs adhered to the scaffolds and formed multilayered cultures. There were numerous filamentous extracellular matrices on the surface of the cells.

A

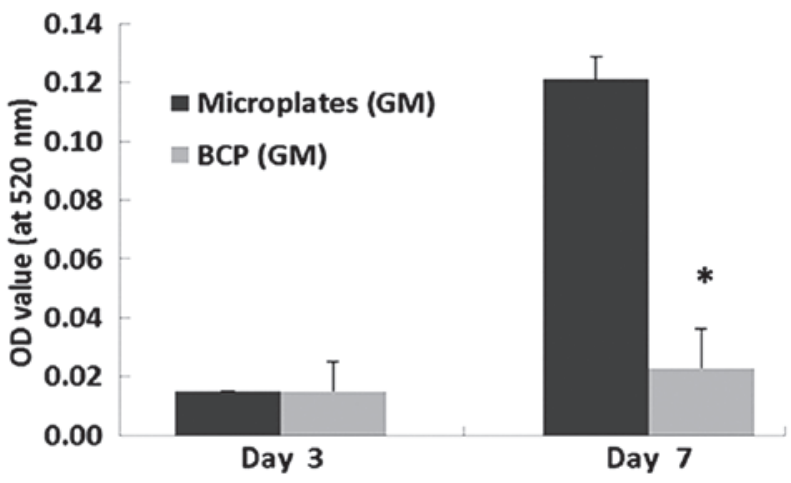

B

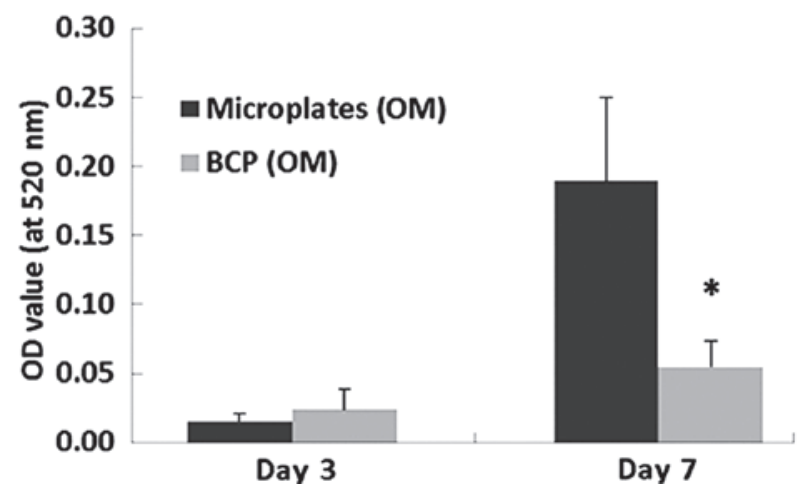

Figure 4. Alkaline phosphatase (ALP) activity of human periodontal ligament cells (hPDLCs) cultured in biphasic calcium phosphate (BCP) scaffolds. (A) In the growth medium (GM), the ALP activity of hPDLCs within BCP scaffolds was comparable with that of the control at day 3; however, it was suppressed significantly at day 7. (B) In the osteogenic medium (OM), ALP activity showed a similar trend. ${ }^{*} \mathrm{P}<0.05$ vs. the control. OD, optical density.

ysis revealed that the BCP scaffolds were able to inhibit the early but not the late proliferation rate of hPDLCs. This inhibitory effect may be associated with the relatively low number of cell adhering to the scaffold surface initially. Another hypothesis to explain this observation is that in a normal physiological bone-formation process, these progenitor cells proliferate rapidly, and the cells progress down the differentiation pathway at the expense of proliferation (19); that is, cells that are differentiating generally exhibit a lower proliferation rate than those that are not $(19,20)$. The present study showed that $\mathrm{BCP}$ significantly induced the expression of OPN mRNA by the hPDLCs in the absence of osteogenic inducers; thus, the cells may have been directed toward osteogenic differentiation causing them to exhibit a low level of proliferation.

ALP activity reflects the early stages of osteogenic differentiation and plays a key role in bone mineralization by initiating and/or promoting the formation of hydroxyapatite crystals in the matrix vesicles of osteoblasts (21). The present study revealed that BCP scaffolds inhibited the ALP activity of hPDLCs. These results conflict with a previous study concerning the behavior of bone cells and dental follicle progenitor cells $(22,23)$. Although the underlying mechanism 
A
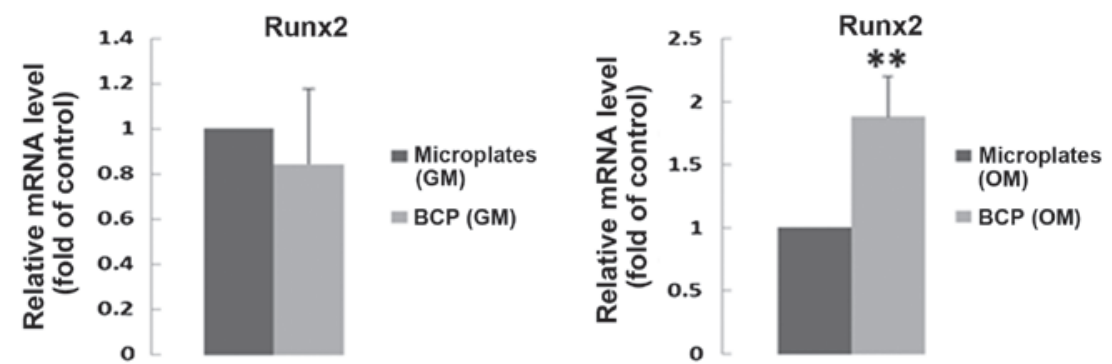

B
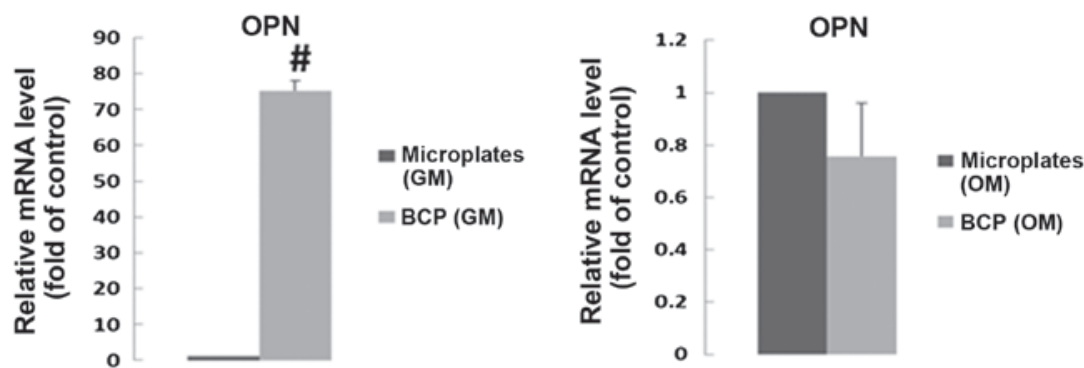

C
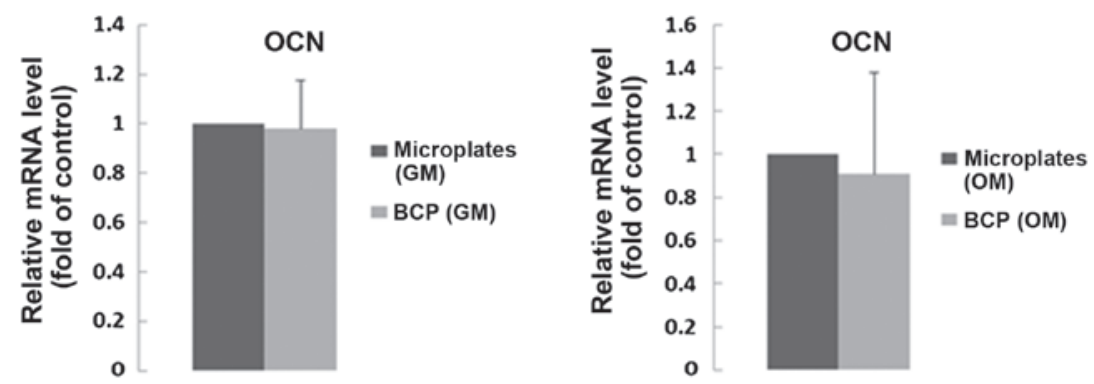

Figure 5. Reverse transcription-quantitative polymerase chain reaction analysis of osteogenic differentiation markers in human periodontal ligament cells (hPDLCs). (A) Runx2, (B) osteopontin (OPN) and (C) osteocalcin (OCN) mRNA. In the growth medium (GM), hPDLCs in biphasic calcium phosphate (BCP) expressed significantly higher levels of OPN compared with the control (monolayer cultures in microplates), and there were no significant differences for Runx 2 and OCN mRNA levels between those cultured in BCP and those in microplates. In the osteogenic medium (OM), Runx2 mRNA levels were significantly higher than those in the control, and OPN and OCN mRNA levels were downregulated slightly. ${ }^{*} \mathrm{P}<0.05,{ }^{* *} \mathrm{P}<0.01,{ }^{*} \mathrm{P}<0.001 \mathrm{vs}$. the control.

for this phenomenon is not clear, a hypothesis is that the degradation of BCP scaffolds results in the dissolution and precipitation of calcium and phosphate ions, which competitively inhibits of ALP $(18,24,25)$.

Runx 2 is the known to be a key regulator of osteoblast marker genes. Runx 2 can also directly stimulate the transcription of osteoblast-related genes such as those encoding OCN and OPN (26). The effect of BCP scaffolds on Runx 2 gene expression differed between the GM and OM. In the OM, BCP scaffolds upregulated Runx 2 mRNA levels, while in the GM, they did not. This result indicates that BCP scaffolds can promote early osteogenic differentiation in the presence of osteogenic inducer (L-aa, Dex and $\beta-G P$ ). However, this significant upregulation of Runx 2 gene expression did not lead to higher levels of OPN and OCN mRNA expression. The lack of a stimulating effect of Runx 2 could be explained by the fact that Runx 2 triggers the expression of major bone matrix genes during the early stages of osteoblast differentiation, but Runx2 is not essential for the maintenance of the expression of these genes in mature osteoblasts and inhibits osteoblast maturation and mature bone formation $(27,28)$.

It has been reported that OPN is necessary for the initiation of hard tissue mineralization (29). It has been also reported that elevated extracellular levels of inorganic phosphate ions upregulate OPN expression in mouse PDL22 cells in a dose-dependent manner (30). BCP scaffolds may release various concentrations of calcium and inorganic phosphate ions depending on the nature of the local microenvironment (composition, $\mathrm{pH}$, temperature and cell type) $(9,24)$. In the present study, hPDLCs within BCP scaffolds expressed significant higher levels of OPN mRNA than the control in the GM. However, in the OM, the OPN mRNA expression levels were unchanged. In the GM, inorganic phosphate ions released from the degradation of BCP scaffolds could affect OPN gene expression in the cell-scaffold co-cultures, whereas the monolayer (microplate) cultures lack this external source of inorganic phosphate ions. In the OM, the $\beta$-GP used to induce mineralization in the osteogenic culture system contains an organic phosphate molecule. Organic phosphate is removed by ALP to release free inorganic phosphate, subsequently providing the chemical potential for the promotion mineral deposition (24). In cell-scaffold co-cultures and monolayer cultures, this released free inorganic phosphate may have an effect. These observations suggested that phosphate ions released from BCP scaffolds may promote OPN gene expression. 
$\mathrm{BCP}$ is biodegradable and is able to release varying quantities of calcium and phosphate ions into the culture medium or a body fluid $(11,12)$. This release of calcium or phosphate ions has a significant impact on the proliferation and osteoblastic differentiation of hPDLCs (16). The results observed in the present study may be derived from the combined effects of the dissolved ions and the scaffold's architecture and surface chemistry.

In conclusion, the results of present study demonstrate that three-dimensional BCP scaffolds are able to induce the early osteoblastic differentiation of hPDLCs in the presence and absence of osteogenic inducer (L-aa, Dex and $\beta-G P$ ). Therefore, BCP scaffolds may be used as effective templates for guiding the attachment, proliferation and osteoblastic differentiation of hPDLCs in vitro.

\section{Acknowledgements}

This study was supported by Guangdong Natural Science Foundation (Grant No. S2012040007041), and Guangdong Science and Technology Planning Program Fund (Grant No. 2013B022000039).

\section{References}

1. Pihlstrom BL, Michalowicz BS and Johnson NW: Periodontal diseases. Lancet 366: 1809-1820, 2005.

2. Monsarrat P, Vergnes JN, Nabet C, Sixou M, Snead ML, Planat-Bénard V, Casteilla L and Kémoun P: Concise review: Mesenchymal stromal cells used for periodontal regeneration: A systematic review. Stem Cells Transl Med 3: 768-774, 2014.

3. Shue L, Yufeng Z and Mony U: Biomaterials for periodontal regeneration: A review of ceramics and polymers. Biomatter 2: 271-277, 2012.

4. Kim JH, Park CH, Perez RA, Lee HY, Jang JH, Lee HH, Wall IB, Shi S and Kim HW: Advanced biomatrix designs for regenerative therapy of periodontal tissues. J Dent Res 93: 1203-1211, 2014.

5. Ramseier CA, Rasperini G, Batia S and Giannobile WV: Advanced reconstructive technologies for periodontal tissue repair. Periodontol 2000 59: 185-202, 2012.

6. Lin NH, Gronthos S and Bartold PM: Stem cells and future periodontal regeneration. Periodontol 2000 51: 239-251, 2009.

7. Bartold PM, McCulloch CA, Narayanan AS and Pitaru S: Tissue engineering: A new paradigm for periodontal regeneration based on molecular and cell biology. Periodontol 2000 24: 253-269, 2000.

8. Taba M Jr, Jin Q, Sugai JV and Giannobile WV: Current concepts in periodontal bioengineering. Orthod Craniofac Res 8: 292-302, 2005.

9. Barrère F, van Blitterswijk CA and de Groot K: Bone regeneration: Molecular and cellular interactions with calcium phosphate ceramics. Int J Nanomedicine 1: 317-332, 2006.

10. El-Ghannam A: Bone reconstruction: From bioceramics to tissue engineering. Expert Rev Med Devices 2: 87-101, 2005.

11. Szpalski C, Wetterau M, Barr J and Warren SM: Bone tissue engineering: Current strategies and techniques - part I: Scaffolds. Tissue Eng Part B Rev 18: 246-257, 2012.

12. Kamitakahara M, Ohtsuki C and Miyazaki T: Review paper: Behavior of ceramic biomaterials derived from tricalcium phosphate in physiological condition. J Biomater Appl 23 197-212, 2008.
13. LeGeros RZ: Properties of osteoconductive biomaterials: Calcium phosphates. Clin Orthop Relat Res 395: 81-98, 2002

14. Yuan $\mathrm{H}$, van Blitterswijk CA, de Groot $\mathrm{K}$ and de Bruijn JD: Cross-species comparison of ectopic bone formation in biphasic calcium phosphate (BCP) and hydroxyapatite (HA) scaffolds. Tissue Eng 12: 1607-1615, 2006.

15. Yao J, Li X, Bao C, Zhang C, Chen Z, Fan H and Zhang X: Ectopic bone formation in adipose-derived stromal cell-seeded osteoinductive calcium phosphate scaffolds. J Biomater Appl 24: 607-624, 2010.

16. Jaiswal N, Haynesworth SE, Caplan AI and Bruder SP: Osteogenic differentiation of purified, culture-expanded human mesenchymal stem cells in vitro. J Cell Biochem 64: 295-312, 1997.

17. Kim H, Kim HW and Suh H: Sustained release of ascorbate-2-phosphate and dexamethasone from porous PLGA scaffolds for bone tissue engineering using mesenchymal stem cells. Biomaterials 24: 4671-4679, 2003.

18. An S, Ling J, Gao Y and Xiao Y: Effects of varied ionic calcium and phosphate on the proliferation, osteogenic differentiation and mineralization of human periodontal ligament cells in vitro. J Periodont Res 47: 374-382, 2012.

19. Holtorf HL, Jansen JA and Mikos AG: Flow perfusion culture induces the osteoblastic differentiation of marrow stroma cell-scaffold constructs in the absence of dexamethasone. J Biomed Mater Res A 72: 326-334, 2005.

20. Lu H, Kawazoe N, Tateishi T, Chen G, Jin X and Chang J: In vitro proliferation and osteogenic differentiation of human bone marrow-derived mesenchymal stem cells cultured with hardystonite $\left(\mathrm{Ca}_{2} \mathrm{ZnSi}_{2} \mathrm{O}_{7}\right)$ and $\{$ beta\}-TCP ceramics. J Biomater Appl 25: 39-56, 2010.

21. Orimo $\mathrm{H}$ and Shimada T: The role of tissue-nonspecific alkaline phosphatase in the phosphate-induced activation of alkaline phosphatase and mineralization in SaOS-2 human osteoblast-like cells. Mol Cell Biochem 315: 51-60, 2008.

22. Bernhardt A, Lode A, Peters F and Gelinsky M: Optimization of culture conditions for osteogenically-induced mesenchymal stem cells in $\beta$-tricalcium phosphate ceramics with large interconnected channels. J Tissue Eng Regen Med 5: 444-453, 2011.

23. Xu LL, Liu HC, Wang DS, E LL, Xu L, Jin ZL and Duan YZ: Effects of BMP-2 and dexamethasone on osteogenic differentiation of rat dental follicle progenitor cells seeded on three-dimensional beta-TCP. Biomed Mater 4: 065010, 2009.

24. Coburn SP, Mahuren JD, Jain M, Zubovic Y and Wortsman J: Alkaline phosphatase (EC 3.1.3.1) in serum is inhibited by physiological concentrations of inorganic phosphate. J Clin Endocrinol Metab 83: 3951-3957, 1998.

25. An S, Gao Y, Ling J, Wei X and Xiao Y: Calcium ions promote osteogenic differentiation and mineralization of human dental pulp cells: Implications for pulp capping materials. J Mater Sci Mater Med 23: 789-795, 2012.

26. Song YG and Cho IH: Characteristics and osteogenic effect of zirconia porous scaffold coated with $\beta$-TCP/HA. J Adv Prosthodont 6: 285-294, 2014.

27. Komori T: Regulation of osteoblast differentiation by Runx 2 . Adv Exp Med Biol 658: 43-49, 2010.

28. Zhang Y, Xie RL, Croce CM, Stein JL, Lian JB, van Wijnen AJ and Stein GS: A program of microRNAs controls osteogenic lineage progression by targeting transcription factor Runx2. Proc Natl Acad Sci USA 108: 9863-9868, 2011.

29. Qin C, Baba O and Butler WT: Post-translational modifications of sibling proteins and their roles in osteogenesis and dentinogenesis. Crit Rev Oral Biol Med 15: 126-136, 2004.

30. Terashima Y, Shimabukuro Y, Terashima H, Ozasa M, Terakura M, Ikezawa K, Hashikawa T, Takedachi M, Oohara H, Yamada S and Murakami S: Fibroblast growth factor-2 regulates expression of osteopontin in periodontal ligament cells. J Cell Physiol 216: 640-650, 2008. 